\title{
Usage and Results of Levosimendan in Ischemic Mitral Valve Surgery
}

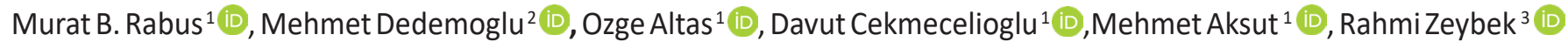 \\ ${ }^{1}$ Kartal Kosuyolu Higher Specialization Training and Research Hospital, Cardiovascular Surgery Department, Istanbul, Turkey. \\ ${ }^{2}$ Mersin Sehir Training and Research Hospital, Cardiovascular Surgery Department, Mersin, Turkey. \\ ${ }^{3}$ Bezm-i Alem University Faculty of Medicine, Cardiovascular Surgery Department, Istanbul, Turkey. \\ Correspondence Author: Murat Bulent Rabus \\ E-mail: muratrabus@yahoo.com
}

Received: $18.08 .2018 \quad$ Accepted: 08.12 .2018

\begin{abstract}
Objective: Large number of comprehensive studies has been carried out on levosimendan. There are many studies on its use, especially in cardiac dysfunction, ischemic cardiac surgery, and heart transplantation surgery. But, there are limited number of studies regarding its use in mitral valve interventions and ischemic mitral dysfunction combined with coronary artery by-pass surgery (CABG). We aimed to investigate the efficacy of levosimendan usage on patients undergone combined coronary artery by-pass grafting and mitral valve surgery because of ischemic mitral dysfunction.
\end{abstract}

Methods: Subsequent patients, who have undergone concurrent CABG and mitral valve repair surgery by a single surgery team, were retrospectively examined. 36 patients were divided into 2 groups; Group 1 (levosimendan therapy group, $n=15$ ) and Group 2 ( $n=21$ ).

Results: There was no statistically significant difference between the groups in terms of preoperative characteristics, echocardiographic data of the patients and preoperative medication. Inotrope therapy was required for 12 patients in Group 1, which was statistically higher than Group 2 ( $n=5, p=0.001$ ). Moreover, IABP following LCOS utilized six and two patients in group 1 and 2 with a statistically significant difference ( $p=0.03)$, respectively.

Conclusion: We recommend using levosimendan on selected patients for its several beneficial effects. However, we do not satisfied with the treatment because the pathology of all patients was not related to ischemia, more to the alteration of ventricle anatomy with deterioration of diameters.

Keywords: Levosimendan, ischemic, mitral valve, mitral repair

\section{INTRODUCTION}

With its combined inotropic and vasodilatory effect, levosimendan having gradually increasing use shows positive effects in treatment of cardiac failure and ventricle dysfunction (1). This medication binds to troponin-C depending on the calcium concentration, improves the myocardial contractility by increasing the calcium-sensitivity of myofilaments, and ensures the peripheral and coronary vasodilation by opening the ATP-sensitive potassium channels $(2,3)$. Thus, both of preload and afterload decrease, coronary blood circulation increases, and anti-ischemic effect appears. Besides that, especially in ischemic cardiac diseases, it plays important role from the aspect of cardiac protection by increasing preoperative and postoperative cardiac output through preoperative myocardial preconditioning.

Large number of comprehensive studies has been carried out on levosimendan. There are many studies on its use, especially in cardiac dysfunction, ischemic cardiac surgery, and heart transplantation surgery. In these studies, they emphasize that levosimendan using before cardiac surgery effected ventricular function positively. But, there are limited number of studies regarding its use in mitral valve interventions and ischemic mitral dysfunction combined with coronary artery by-pass surgery (CABG). However, studies investigating the efficacy of levosimendan for ventricular function in patients with ischemic mitral regurgitation are limited. In this regard, we aimed to investigate the effect of preoperative levosimendan administration on ventricular function in patients with ischemic mitral regurgitation. Hence, we aimed to investigate the use of levosimendan on patients undergone combined coronary artery by-pass grafting and mitral repair surgery due to coronary cardiac disease and ischemic mitral dysfunction. 


\section{MATERIAL AND METHODS}

Subsequent patients, who have undergone concurrent CABG and mitral valve repair surgery by a single surgery team, were retrospectively examined. This study was reviewed and approved by the Kartal Kosuyolu Higher Specialization Research and Training Hospital Ethics Committee for NonInvasive Clinical Research (Reg. No: 2020.2/08-293). The inclusion criteria were coronary artery disease, severe left ventricle dysfunction and moderate left ventricle dysfunction having increased ventricle diameter, and onpump CABG and mitral repair surgery; whereas, exclusion criteria were previous cardiac surgery, tricuspid non-valve pathologies, renal and liver dysfunction, preoperative intubation, emergency cardiac surgery interventions and valve replacement requirements. 16 patients having these characteristics were excluded from the study. 36 patients were divided into 2 groups; patients with low ventricular function, enlarged ventricular diameter and levosimendan retrospectively were identified as group 1 . Group 1 (levosimendan therapy group, $\mathrm{n}=15$ ) and patients with the same characteristics and no levosimendan were identified as group 2. Group $2(n=21)$. Preoperative characteristics of patients were summarized in Table 1 (Table 1). Each patient was examined echocardiographically prior to operation (Table 2).

Table 1. Preoperative demographic characteristics of patients.

\begin{tabular}{|l|c|c|c|}
\hline & Group 1 (n=15) & Group 2 (n=21) & P value \\
\hline Age (years) & $57.3 \pm 12$ & $62.8 \pm 7.8$ & 0.1 \\
\hline Male & $11(73 \%)$ & $14(66 \%)$ & 0.6 \\
\hline Weight $(\mathrm{kg})$ & $66.86 \pm 14.35$ & $73.42 \pm 14.53$ & 0.3 \\
\hline Height $(\mathrm{cm})$ & $161.7 \pm 10.6$ & $160.1 \pm 8.7$ & 0.6 \\
\hline BSA (m²) & $1.73 \pm 0.2$ & $1.8 \pm 0.2$ & 0.3 \\
\hline COPD & $2(13 \%)$ & $3(14 \%)$ & 0.6 \\
\hline Diabetes & $9(60 \%)$ & $7(33 \%)$ & 0.11 \\
\hline Unstable angina & $1(7 \%)$ & $3(14 \%)$ & 0.47 \\
\hline Euroscore & $7.91 \pm 6.50$ & $11.88 \pm 5.08$ & 0.6 \\
\hline
\end{tabular}

BSA: body surface area; COPD: Chronic Obstructive Pulmonary Disease

The patients in Group 1 were taken into intensive care unit 24 hours before the surgical intervention, and levosimendan therapy was initiated preoperatively $(200 \mathrm{mcg} / \mathrm{kg})$ under the control of heart rate, arterial blood pressure, central venous pressure and pulse oximeter with hourly urine analysis. Moreover, the treatment was continued during the operation following to postoperative 24 hours.
Table 2. Preoperative echocardiographic evaluation of the patients

\begin{tabular}{|l|c|c|c|}
\hline & Group 1 (n=15) & Group 2 (n=21) & P value \\
\hline PAP $(\mathrm{mmHg})$ & $65.18 \pm 32.51$ & $51.75 \pm 13.40$ & 0.11 \\
\hline LVESD $(\mathrm{cm})$ & $4.8 \pm 0.7$ & $4.25 \pm 0.85$ & 0.06 \\
\hline LVEDD $(\mathrm{cm})$ & $6.11 \pm 0.72$ & $5.73 \pm 0.70$ & 0.13 \\
\hline LEDV $\left(\mathrm{ml} / \mathrm{m}^{2}\right)$ & $185.43 \pm 55.90$ & $174.62 \pm 51.41$ & 0.67 \\
\hline LESV $\left(\mathrm{ml} / \mathrm{m}^{2}\right)$ & $112.65 \pm 39.06$ & $87.75 \pm 43.29$ & 0.21 \\
\hline LA (cm) & $4.56 \pm 1.19$ & $4.62 \pm 0.72$ & 0.62 \\
\hline Posterior wall (cm) & $0.97 \pm 0.21$ & $1.05 \pm 0.12$ & 0.19 \\
\hline IVS (cm) & $0.99 \pm 0.20$ & $1.08 \pm 0.13$ & 0.12 \\
\hline LVEF $(\%)$ & $34.80 \pm 6.96$ & $34.76 \pm 6.22$ & 0.58 \\
\hline MI (grade) & & & \\
\hline $3 / 4$ & $7(47 \%)$ & $9(43 \%)$ & \\
\hline $4 / 4$ & $8(53 \%)$ & $12(57 \%)$ & \\
\hline TI (grade) & $3(20 \%)$ & $4(19 \%)$ & \\
\hline $2 / 4$ & $2(13 \%)$ & 0 & \\
\hline $3 / 4$ & $1(6 \%)$ & $1(5 \%)$ & \\
\hline $4 / 4$ & & & \\
\hline IVS: & & & \\
\hline
\end{tabular}

IVS: interventricular septum; LA: left atrium; LEDV: left ventricular enddiastolic volume; LESV: left ventricular end-systolic volume; LVEDD: left ventricular end diastolic diameter; LVEF: left ventricular ejection fraction; LVESD: left ventricular end systolic diameter; MI: mitral insufficiency, PAP: pulmonary artery pressure; TI: tricuspid insufficiency

\subsection{Operation-Surgical Method}

All of the patients were intubated under general anesthesia (fentanyl $5 \mathrm{mcg} / \mathrm{kg}$, midazolam $0.1 \mathrm{mg} / \mathrm{kg}$, vecuronium bromide $0.15 \mathrm{mg} / \mathrm{kg}$, and propofol $1 \mathrm{mg} / \mathrm{kg}$ ). Inhaled sevoflurane with fentanyl and propofol infusions $(1 \mathrm{mg} / \mathrm{kg} /$ hour) were implemented.

Standard aortic arterial and bi-caval venous cannulation was utilized for cardiopulmonary by-pass (CPB). Myocardial protection with mild hypothermia was achieved via intermittent anterograde cardioplegia, whereas continuous retrograde cardioplegia was also preferred in some patients. Following the cross-clamp, distal anastomoses, mitral valve repair and proximal anastomoses were performed respectively. Ring annuloplasty is the common method for all patients undergoing repair.

Preoperative basal creatinine level higher than 50\% and/ or anuria was considered to be renal, suddenly-developing stroke or temporary ischemic attack to be neurological, respiratory dysfunction and repetitive mechanic ventilation need to be respiratory; any tachycardia, bradycardia, atrial and ventricular dysrhythmias to be arrhythmia and hemorrhage $\geq 500 \mathrm{ml}$ at $1^{\text {st }}$ postoperative hour, $\geq 400 \mathrm{ml}$ at $2^{\text {nd }}$ postoperative hour, and $\geq 300 \mathrm{ml}$ at $3^{\text {rd }}$ postoperative hour were considered to be hemorrhagic complications. Patients having systolic arterial pressure of $<80 \mathrm{mmHg}$, diastolic arterial pressure of $<50 \mathrm{mmHg}$ and not responding to liquid replacement treatment were given inotropic therapy. Hypotension, high central venous pressure, tachycardia, sweating, oliguria, and severe ventricular dysfunction in echocardiographic examination were considered as low 
cardiac output syndrome, in whom intraaortic balloon pump (IABP) support was applied.

\subsection{Statistical Analysis}

Statistical analyses were performed using Statistical Package for the Social Sciences (SPSS software Version 15.0, IBM Analytics, New York, USA). The normal distribution of variables was examined using visual (histogram and probability graphs) and analytical methods (Kolmogorov-Smirnov/Shapiro-Wilk tests). Descriptive analyses were performed using frequency tables for categorical variables, and mean and standard deviation for normally-distributed variables. Median and interquartile ranges were used for non-normally distributed variables. Independent sample t-test was used for normally distributed variables; whereas, Mann-Whitney $U$ test for non-normally distributed variables, and Pearson Chi-Square test for categorical variables between two groups. The Paired t-test and Wilcoxon test were utilized for dependent groups. $\mathrm{p}<0.05$ was considered statistically significant.

\section{RESULTS}

There was no statistically significant difference between the groups in terms of preoperative characteristics, echocardiographic data of the patients (Tables 1 and 2) and preoperative medication (Table 3).

Table 3. Preoperative medication management

\begin{tabular}{|l|c|c|c|}
\hline & Group 1 (n=15) & Group 2 (n=21) & P value \\
\hline Nitrates & $12(80 \%)$ & $18(86 \%)$ & 0.65 \\
\hline ACE inh/ARB & $9(60 \%)$ & $9(43 \%)$ & 0.31 \\
\hline Beta Blockers & $5(33 \%)$ & $3(14 \%)$ & 0.17 \\
\hline Diuretics & $7(47 \%)$ & $5(24 \%)$ & 0.15 \\
\hline
\end{tabular}

$A C E$ inh: angiotensin converting enzyme inhibitor; $A R B$ : angiotensin receptor blocker

Given the intraoperative data, the levels of hypothermia were similar in both groups $(p=0.85)$. In addition, there was no statistically significant difference regarding CPB and crossclamp time ( $p=0.34$ and 0.06 , respectively). Continuous retrograde cardioplegia was added to 29 patients (80\%) with no statistical difference in terms of cardioplegia strategies. Left internal mammary artery (LIMA) was the choice of left anterior descending (LAD) anastomoses; vein graft was used for other graft anastomoses. Furthermore, various reconstruction methods, such as Alfieri procedure $(n=2)$, McCune plasty $(n=2)$, neochorda implantation $(n=1)$ and posterior leaflet plication $(n=12)$, were employed for repairing in addition to ring annuloplasty performed to all patients. Additional Tricuspid valve repair was employed for 5 patients in Group 1 and 6 patients in Group 2, whereas there was no statistically significant difference between the groups (Table 4).
Table 4. Operative values of the patients

\begin{tabular}{|c|c|c|c|}
\hline & Group 1 ( $n=15)$ & Group 2 ( $n=21$ ) & $P$ value \\
\hline Hypothermia $\left({ }^{\circ} \mathrm{C}\right)$ & $31.56 \pm 1.66$ & $31.35 \pm 2.98$ & 0.85 \\
\hline ACC time (min) & $89.20 \pm 25.27$ & $100.61 \pm 34.95$ & 0.06 \\
\hline CPB time (min) & $132.86 \pm 33.09$ & $143.80 \pm 34.38$ & 0.34 \\
\hline Retrograde cardioplegia & $10(67 \%)$ & $19(90 \%)$ & 0.07 \\
\hline \multicolumn{4}{|l|}{ Number of grafts } \\
\hline 1 & $6(40 \%)$ & $7(33 \%)$ & \\
\hline 2 & $7(47 \%)$ & $9(43 \%)$ & \\
\hline 3 & $2(13 \%)$ & $5(24 \%)$ & \\
\hline $\begin{array}{l}\text { Concomitant tricuspid } \\
\text { reconstruction }\end{array}$ & $6(40 \%)$ & $5(24 \%)$ & 0.46 \\
\hline
\end{tabular}

ACC: aortic cross clamping; CPB: cardiopulmonary bypass.

Given the postoperative data of patients, as a neurological complication, temporary ischemic attack was observed in one patient (Group 1). The renal problems were observed in seven patients (four in Group 1 and three patients in Group 2, $p=0.35$ ) with temporary hemodialysis in two and hemofiltration in one. Renal replacement treatment was utilized for the rest of the patients. There was no statistically significant difference between the groups in these findings. Respiratory complications were observed in one patient in Group 1 and seven patients in Group 2; hence, there was statistically significant difference between groups $(p=0.05)$. Postoperative arrhythmia was observed in nine patients in Group 1 and 11 patients in Group $2(p=0.2)$. Atrial fibrillation needed medication, temporary AV block and nodal rhythm was observed in five, four and two patients, respectively. Permanent pacing was required in only one patient. There was no statistically significant difference between the groups in these findings. Vasopressor and inotropic use were present in 6 and 11 patients in group 1 and 5 and 12 patients in group 2, respectively, and there was no statistical difference between vasopressor and inotropic use between groups. Looking at the use of IABP; the use of IABP was observed in 6 patients in group 1 and in 2 patients in group 2 and the difference between the groups was statistically significant (p:0.03). Revision for postoperative bleeding was observed in one patient in Group 1. Hospital mortality was seen in two patients of group 1 (Table 5). Longer duration of ventilation and hospitalization in ICU were observed in group 1 when compared to group 2. ( $p=0.01$ and $p=0.003)$. However, there was no statistically significant difference $(p=0.11)$ regarding hospital stay (Table 6).

There was no statistically significant difference in terms of echocardiographic data in two groups. Residual mitral regurgitation was observed in two patients in Group 1 and in three patients in Group 2 without statistically difference (Table 7).

Decrease in left ventricular systolic and diastolic end diameters and diastolic end volumes were seen in group 1, even though there was no statistically difference comparing pre - and post-operative echocardiographic data. Moreover, increase in posterior wall thickness was significant $(p=0.04)$. Reduction in pulmonary artery pressure was observed (Table 8). 
Table 5. Postoperative adverse events

\begin{tabular}{|l|c|c|c|}
\hline Complications & $\begin{array}{c}\text { Group 1 } \\
(\mathbf{n = 1 5})(\mathbf{n}, \mathbf{\%})\end{array}$ & $\begin{array}{c}\text { Group 2 } \\
(\mathbf{n = 2 1 )}(\mathbf{n}, \mathbf{\%})\end{array}$ & P value \\
\hline Neurologic & $1(7)$ & $0(0)$ & 0.23 \\
\hline Respiratory & $4(26)$ & $2(9)$ & 0.17 \\
\hline Renal & $4(27)$ & $3(14)$ & 0.35 \\
\hline Arrhythmia & $9(60)$ & $11(52)$ & 0.2 \\
\hline Inotropic support & $7(46)$ & $4(19)$ & 0.15 \\
\hline Vasopressor support & $10(66)$ & $7(33)$ & 0.12 \\
\hline IABP support & $6(40)$ & $2(9)$ & $0.03 *$ \\
\hline Hemorrhage & $1(7)$ & $0(0)$ & 0.23 \\
\hline Infection & $2(13)$ & $0(0)$ & 0.08 \\
\hline Mortality & $2(13)$ & $0(0)$ & 0.08 \\
\hline
\end{tabular}

IABP: intraaortic balloon pump

Table 6. The duration of ventilation, ICU and hospital stay

\begin{tabular}{|l|c|c|c|}
\hline & $\begin{array}{c}\text { Group 1 } \\
\text { (n:15) }\end{array}$ & $\begin{array}{c}\text { Group 2 } \\
\text { (n:21) }\end{array}$ & $\begin{array}{c}\text { P } \\
\text { value }\end{array}$ \\
\hline Duration of ventilation (hour) & $43.13 \pm 38.76$ & $24.66 \pm 36.38$ & $0.01^{*}$ \\
\hline Length of ICU (day) & $8.60 \pm 5.97$ & $4.19 \pm 2.82$ & $0.003^{*}$ \\
\hline Length of hospitalization (day) & $13.72 \pm 5.60$ & $12.52 \pm 6.17$ & 0.11 \\
\hline
\end{tabular}

ICU: Intensive care unit

Table 7. Postoperative echocardiographic evaluation of the patients

\begin{tabular}{|l|c|c|c|}
\hline & Group 1 $(\mathbf{n = 1 5})$ & Group 2 $(\mathrm{n}=\mathbf{2 1})$ & P value \\
\hline LVESD $(\mathrm{cm})$ & $4.50 \pm 0.55$ & $4.27 \pm 0.88$ & 0.57 \\
\hline LVEDD $(\mathrm{cm})$ & $5.75 \pm 0.44$ & $5.52 \pm 0.72$ & 0.48 \\
\hline LEDV $\left(\mathrm{ml} / \mathrm{m}^{2}\right)$ & $168 \pm 36.09$ & $142.78 \pm 47.09$ & 0.4 \\
\hline LESV $\left(\mathrm{ml} / \mathrm{m}^{2}\right)$ & $94.36 \pm 31.54$ & $88.14 \pm 39.01$ & 0.8 \\
\hline LA $(\mathrm{cm})$ & $4.66 \pm 0.62$ & $4.68 \pm 0.73$ & 0.89 \\
\hline Posterior wall $(\mathrm{cm})$ & $1.10 \pm 0.10$ & $1.04 \pm 0.10$ & 0.30 \\
\hline IVS $(\mathrm{cm})$ & $1.10 \pm 0.15$ & $1.11 \pm 0.15$ & 0.83 \\
\hline LVEF $(\%)$ & $36.87 \pm 7.98$ & $39.47 \pm 7.05$ & 0.48 \\
\hline MI (grade) & & & \\
\hline 2/4 & $2(5 \%)$ & $3(8 \%)$ & 0.9 \\
\hline
\end{tabular}

IVS: interventricular septum; LA: left atrium; LEDV: left ventricular enddiastolic volume; LESV: left ventricular end-systolic volume; LVEDD: left ventricular end diastolic diameter; LVEF: left ventricular ejection fraction; LVESD: left ventricular end systolic diameter; MI: mitral insufficiency

Table 8. Pre - and post-operative echocardiographic comparison in patients who received levosimendan

\begin{tabular}{|l|c|c|c|}
\hline & Preoperative TTE & Postoperative TTE & P value \\
\hline LVEF $(\%)$ & $34.80 \pm 6.96$ & $36.87 \pm 7.98$ & 0.28 \\
\hline LVESD $(\mathrm{cm})$ & $4.8 \pm 0.7$ & $4.50 \pm 0.55$ & 0.07 \\
\hline LVEDD $(\mathrm{cm})$ & $6.11 \pm 0.72$ & $5.75 \pm 0.44$ & 0.11 \\
\hline LA $(\mathrm{cm})$ & $4.56 \pm 1.19$ & $4.66 \pm 0.62$ & 0.24 \\
\hline IVS $(\mathrm{cm})$ & $0.99 \pm 0.20$ & $1.10 \pm 0.15$ & 0.1 \\
\hline Posterior wall $(\mathrm{cm})$ & $0.97 \pm 0.21$ & $1.10 \pm 0.1$ & $0.04^{*}$ \\
\hline PAP $(\mathrm{mmHg})$ & $65.18 \pm 32.51$ & $55.0 \pm 32.01$ & 0.14 \\
\hline LESV $\left(\mathrm{ml} / \mathrm{m}^{2}\right)$ & $112.65 \pm 39.06$ & $94.36 \pm 31.54$ & 0.18 \\
\hline LEDV $\left(\mathrm{ml} / \mathrm{m}^{2}\right)$ & $185.43 \pm 55.90$ & $168 \pm 36.09$ & 0.1 \\
\hline
\end{tabular}

IVS: interventricular septum, LA: left atrium, LEDV: left ventricular enddiastolic volume, LESV: left ventricular end-systolic volume, LVEDD: left ventricular end diastolic diameter, LVEF: left ventricular ejection fraction, LVESD: left ventricular end systolic diameter, PAP: pulmonary artery pressure, TTE: Trans-thoracic echocardiography

\section{DISCUSSION}

Levosimendan is a positive inotropic and calcium-sensitizer agent supporting diastolic function of the heart (4). Moreover, showing effects on ATP-sensitive potassium channels on the mitochondria, it preconditions the myocardium against ischemia and has a protective mechanism against the ischemia reperfusion damage (5). Without disturbing the energy balance of the heart, it creates positive inotropic effects. It also increases the myocardial contractility and cardiac output without increasing the oxygen demand of myocardia (6). Short-term infusion of levosimendan allows lower level of damage on dysfunctional myocardia and creates the preconditioning effect $(7,8)$. Besides, it has effects on improving the cardiac index and mean arterial pressure and decreasing the pulmonary capillary wedge pressure (6-9). In our study, even if the results are not statistically significant, reduction was observed both in left ventricle systolic/diastolic end diameters and volumes. There was also a statistically significant increase in left ventricular posterior wall thickness. Moreover, an improvement was observed in pulmonary arterial pressures, which have been high during preoperative period.

In current guidelines, levosimendan referred as Class 2A from inotropic aspect that having proof level C (10), is used in various stages of cardiac surgery. In many reports, levosimendan is used in weaning from CPB or in postoperative period, and even before the surgery (8). We preferred to initiate 24 hours before the surgery, and then continued for 24 hours after the operation.

However, in some studies, the half-life of levosimendan was reported to be one hour but that of the active metabolite to be approx. 80 hours and, for this reason, the effect continues in early period even if the treatment was stopped $(6,11)$.

One of the issues emphasizing on levosimendan is whether it is inducing higher doses of inotoropic agents. And, yet, there is still much controversy between the studies on this topic.

It was asserted in some of the studies, in which it decreases the use of inotrope, meanwhile the others have been reported with a reduction in the high-dose inotrope $(11,12)$. On the contrary, some has reported an increasing effect of inotrope and vasopressin usage (9). In our study, the increase in the use of additional inotropes was not statistically significant in patients using levosimendan. As a matter of fact, it is obvious to use vasopressor agents due to the hypotensive effect of levosimendan. On the other hand, according to guidelines, it is recommended to not use it unless it is combined with inotrope and vasopressor agents in patients either with hypotension (systolic blood pressure of $<85 \mathrm{mmHg}$ ) or in cardiogenic shock (10). However, the use of vasopressor in the group receiving levosimendan for the prevention of possible hypotension effect in our study, however, was not statistically significant. Kolseth et al. determined that the prophylactic use of levosimendan in patients with reduced left ventricular ejection fraction(LVEF) has no superiority over the catecholamines (13). 
Even though levosimendan is said to have been reduced IABP initiation in some studies (11), we observed an increase in group 1, astonishingly as not expected. Another remarkable issue is the effects on ventilation and the length of intensive care unit (ICU) and hospital stay. There are some studies depicting the reduction in all parameters, and some are not $(7-9,12)$. There was no statistically significant difference between the groups in the duration of hospital stay in our study. Although there is a statistically significant difference in intensive care unit stay in the levosimendan group, we do not think it is clinically significant. Because patients receiving levosimendan were admitted to intensive care for drug usage preoperatively. Adverse effects, such as nausea, vomiting, hypotension, can be seen with the higher doses (9). However, Lahtinen et al. came up with a decrease hemorrhage with levosimendan after valve surgery (14). In our study, there was no statistically significant difference between the groups in terms of postoperative complications.

Regarding the effect of levosimendan on mortality, the common opinion showed no effect on mortality and morbidity $(8,13)$. In our study, mortality was observed in two patients, whom were in group 1, due to the sepsis. Besides, parallel to publications, there was no difference between groups in terms of early mortality. During the last decade, Alvarez and colleagues brought different dimension and they claimed that proven beneficial effects of levosimendan should not be considered due to the lack of larger sample sizes. And, they said that it is controversial to clarify the increased survival with levosimendan in the absence of randomized studies (15).

\section{CONCLUSION}

The use of levosimendan in cardiac surgery provides many beneficial effects. We conclude that levosimendan has a significant positive effect on cardiac function and ventricular diameters for ischemic mitral valve patients, even if it is not statistically significant. In addition, we do not see any harmful effects. However, since we can not see very great superiority as expected, we think that it will be more beneficial to use it in selected patients from the point of view of cost effectiveness. Finally, since the studies on these groups of patients undergoing combined surgery are limited, larger scale studies are needed.

Acknowledgment: The study and outcomes presented orally during 13th International Congress of Update in Cardiology and Cardiovascular Surgery which held in Izmir, Turkey between 23-26 March, 2017.

\section{REFERENCES}

[1] Slawsky MT, Colucci WS, Gottlieb SS, Greenberg BH, Haeusslein, E, Hare J, Hutchins S, Leier CV, Le Jemtel TH, Loh E, Nicklas
J. Acute hemodynamic and clinical effects of levosimendan in patients with severe heart failure. Circulation, 2000; 102(18):2222-2227.

[2] Lehmann A, Boldt J, Kirchner J. The role of Ca-sensitizers for the treatment of heart failure. Curr Opin Crit Care 2003; 9:337-344.

[3] McBride BF, White CM. Levosimendan: implications for clinicians. J Clin Pharmacol 2003;43:1071-1081.

[4] Meyer K, Schipke JD, Klocke RC, Gams E, Korbmacher B. Inotropic, vasodilating and preconditioning actions of levosimendan in the heart. Thorac Cardiovasc Surg 2008;56(07): 379-385.

[5] Antoniades C, Tousoulis D, Koumallos N, Marinou K, Stefanadis C. Levosimendan: beyond its simple inotropic effect in heart failure. Pharmacol Ther 2007;114:184-197.

[6] Morais RJ. Levosimendan in severe right ventricular failure following mitral valve replacement. J Cardiothorac and Vasc Anesthesia 2006;20:82-84.

[7] Sharma P, Malhotra A, Gandhi S, Garg P, Bishnoi A, Gandhi H. Preoperative levosimendan in ischemic mitral valve repair. Asian Cardiovasc Thorac Ann 2014;22:539-545.

[8] Ersoy O, Boysan E, Unal EU. Effectiveness of prophylactic levosimendan in high-risk valve surgery patients. Cardiovasc J Afr 2013;24:260-264.

[9] Ravikumar G, Syamasundar A, Ravulapalli H, Karthekeyan RB, Vakamudi M, Kodalli R, Nandipati S. A comparison of hemodynamic effects of levosimendan and dobutamine in patients undergoing mitral valve repair / replacement for severe mitral stenosis. Ann Card Anaesth 2013;16:11-15.

[10] Ponikowski P, Voors AA, Anker SD, Bueno H, Cleland JG, Coats AJ, Falk V, González-Juanatey JR, Harjola VP, Jankowska EA, Jessup M. ESC Guidelines for the diagnosis and treatment of acute and chronic heart failure: Pharmacological therapy of acute heart failure. Eur J Heart Fail 2016;18(8):891-975.

[11] Tokuda Y, Grant P, Wolfenden H, Manganas C, Lyon W, Murala J. Levosimendan for patients with impaired left ventricular function undergoing cardiac surgery. Interact Cardiovasc Thorac Surgy 2006;5:322-326.

[12] Tasouli A, Papadopoulos K, Antoniou T, Kriaras I, Stavridis G, Degiannis D, Geroulanos S. Efficacy and safety of perioperative infusion of levosimendan in patients with compromised cardiac function undergoing open-heart surgery: importance of early use. Eur J Cardiothorac Surg 2007;32:629-633.

[13] Kolseth SM, Nordhaug DO, Stenseth R, Sellevold O, KirkebyGarstad I, Wahba A. Prophylactic treatment with levosimendan: a retrospective matched-control study of patients with reduced left ventricular function. Eur J Cardiothorac Surg 2009;36:1024-1030.

[14] Lahtinen P, Pitkänen O, Musialowicz T. Levosimendan increases bleeding risk after heart valve surgery: a retrospective analysis of a randomized trial. J Cardiothorac Vasc Anesth 2014;28:1238-1242.

[15] Álvarez J. Levosimendan and low cardiac output syndrome: does mortality really decrease? Rev Esp Cardiol 2008;61:454-457. 